\title{
Editorial
}

\section{Analysis of Nonlinear Dynamics of Neural Networks}

\author{
Sabri Arik, ${ }^{1}$ Juhyun Park, ${ }^{2}$ Tingwen Huang, ${ }^{3}$ and José J. Oliveira ${ }^{4}$ \\ ${ }^{1}$ Department of Electrical and Electronics Engineering, Isik University, Sile, 34980 Istanbul, Turkey \\ ${ }^{2}$ Nonlinear Dynamics Group, Department of Electrical Engineering, Yeungnam University, Gyeongsan 712-749, Republic of Korea \\ ${ }^{3}$ Science Program, Texas AઐM University at Qatar, P.O. Box 23874, Doha, Qatar \\ ${ }^{4}$ Departamento de Matemática e Aplicações e CMAT, Escola de Ciências, Universidade do Minho, \\ Campus de Gualtar, 4710-057 Braga, Portugal \\ Correspondence should be addressed to Sabri Arik; ariks@isikun.edu.tr
}

Received 7 March 2013; Accepted 7 March 2013

Copyright (C) 2013 Sabri Arik et al. This is an open access article distributed under the Creative Commons Attribution License, which permits unrestricted use, distribution, and reproduction in any medium, provided the original work is properly cited.

Dynamical neural networks proved to be an important tool to solve some practical engineering problems in the areas such as optimization, image and signal processing, control systems, associative memories, and pattern recognition. When employing neural networks to solve practical engineering problems, it is crucial to be able to completely characterize the dynamical properties of neural networks. There are many various classes of neural network models that can be described in the form of nonlinear systems. Therefore, neural networks may exhibit extremely different complex dynamical behaviors depending on the model and network parameters. Hence, the analysis of nonlinear dynamics of neural networks still possesses new challenges to researchers.

The aim of this special issue is to solicit theoretical and application research in the fields of neural networks exploiting their nonlinear dynamics. We believe it provides a good opportunity for reflection on current developments in the nonlinear analysis of dynamical behaviors of neural networks. The papers submitted to this special issue represent a mixture of cross-cutting investigations and provide deep insight into the current developments in the field. The accepted papers in this special issue addressed the following topics:

(i) stability analysis of dynamical neural networks,

(ii) almost periodic solution of of neutral-type neural networks,

(iii) impulsive control of stochastic synchronization of reaction-diffusion neural networks, (iv) neural network model for predicting peak ground acceleration,

(v) dynamical analysis of high-order neural networks,

(vi) synchronization of nonlinear coupled complex networks.

As we mentioned above, the special issue aimed to reveal new ideas in the area of nonlinear dynamics of neural networks, which would be helpful for the scientists and researchers who share the common interest in neural networks. We hope that the readers will agree with us that the published papers reflect convincingly the issue's objectives with its variety of presented topics, investigated at both theoretical and application levels.

Sabri Arik Juhyun Park Tingwen Huang José J. Oliveira 


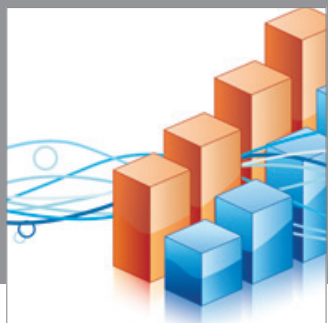

Advances in

Operations Research

mansans

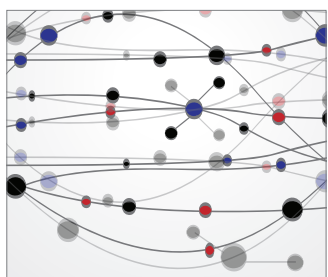

The Scientific World Journal
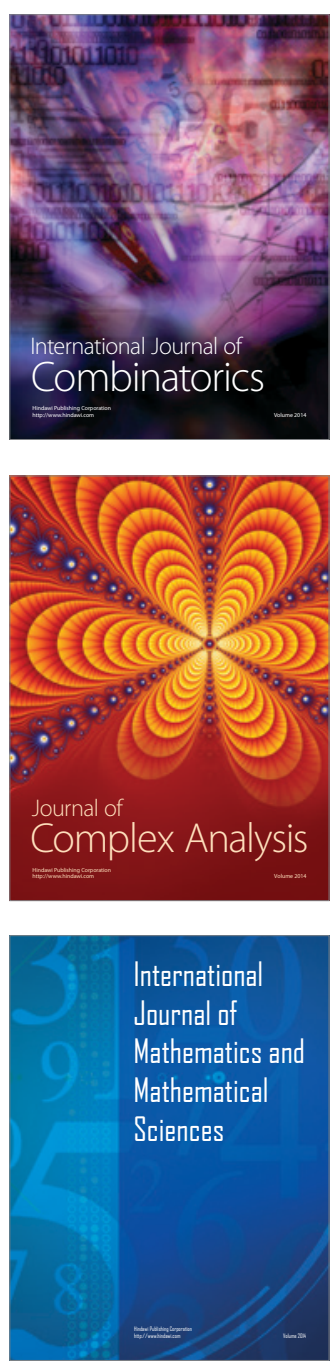
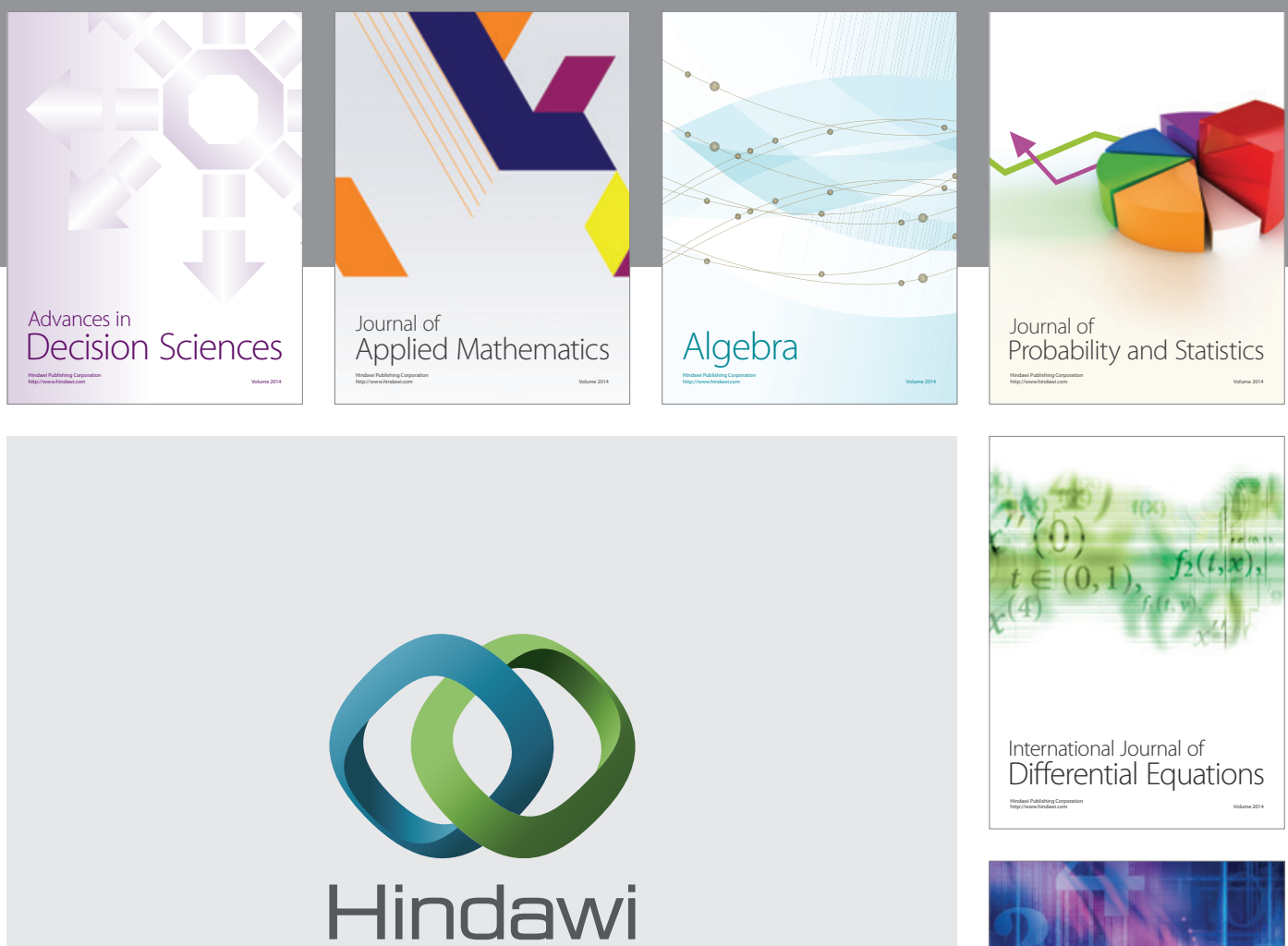

Submit your manuscripts at http://www.hindawi.com
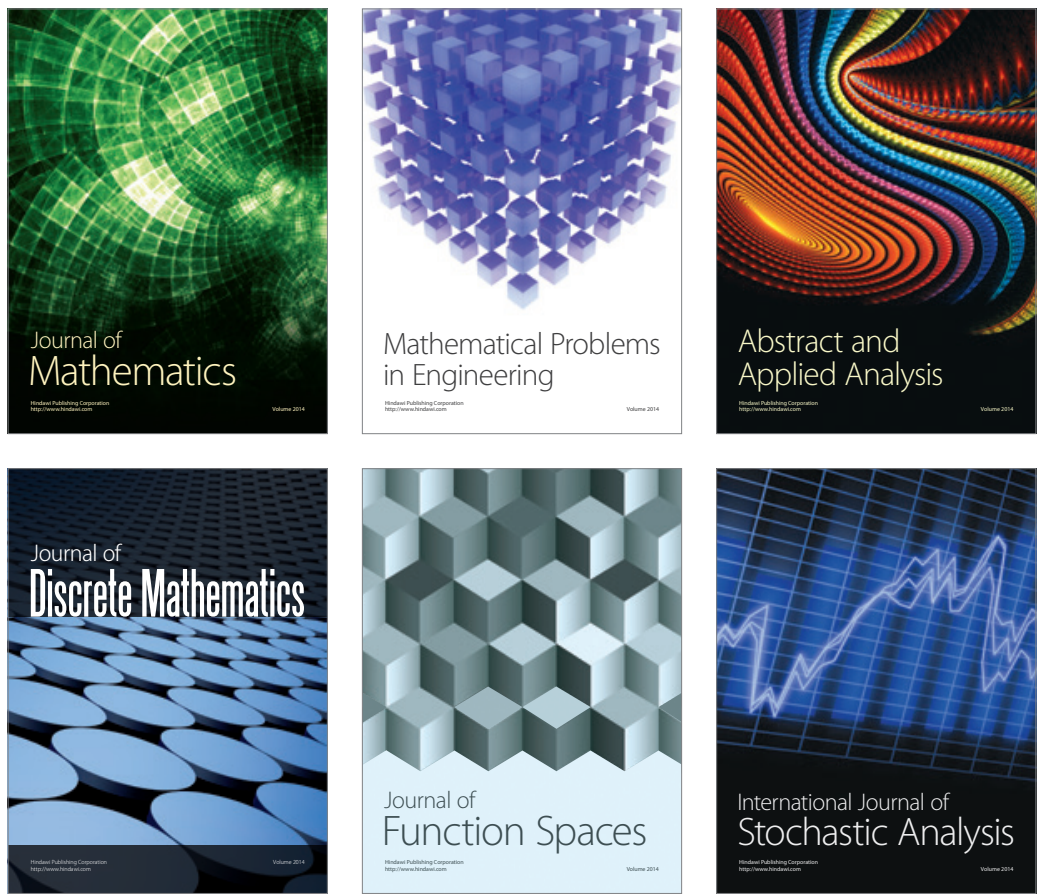

Journal of

Function Spaces

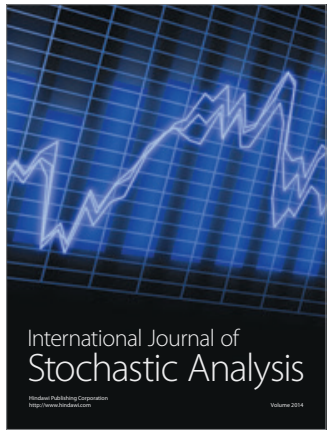

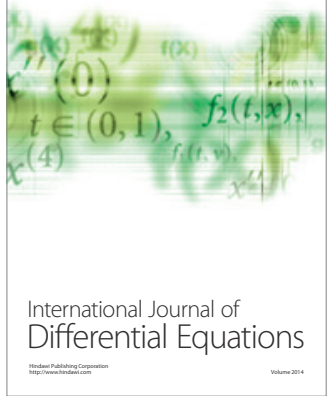
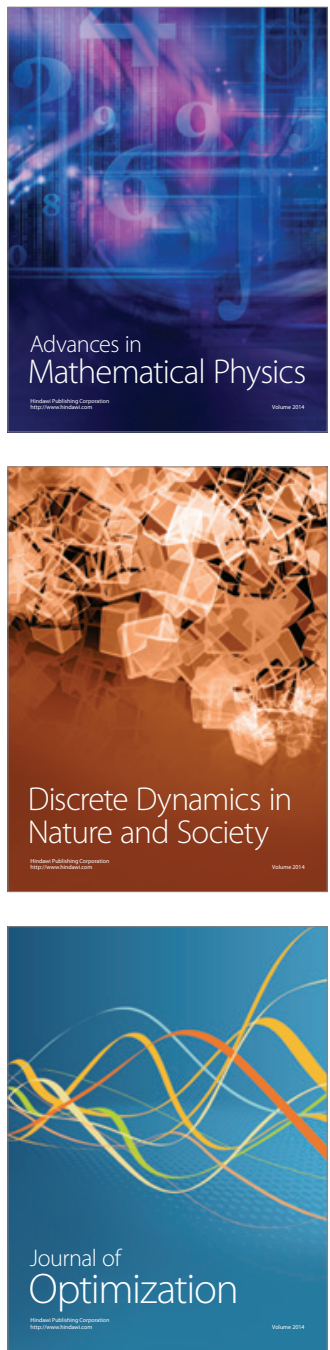